The International Journal Of Engineering And Science (IJES)

|| Volume || 6 || Issue || 1 || Pages || PP 13- 21 || 2017 ||

ISSN (e): $2319-1813 \operatorname{ISSN}$ (p): $2319-1805$

\title{
Contraintes À L’acces Aux Soins De Santé Maternelle Dans La Ville De Maroua
}

\author{
Eloundou Messi ${ }^{1}$, Waïbaï Yaye ${ }^{2}$ \\ ${ }^{I}$ Paul Basile Université de Maroua \\ ${ }^{2}$ HonorineUniversité de Maroua
}

\begin{abstract}
-
L'accès aux soins de santé maternelle dans la ville de Maroua demeure restrictif malgré les grands efforts de création, de construction, d'équipement des formations sanitaires et l'existence d'un document de stratégie nationale de technologie de la santé. En effet, une proportion importante de la population de la ville de Maroua n'a pas accès aux soins de santé essentiels, soit parce que les services adéquats ne sont pas disponibles, soit parce que ces services ne sont pas accessibles à la bourse des plus pauvres. Cette situation est imputable à plusieurs contraintes qui limitent l'accès aux soins de santé accentuant ainsi la mortalité maternelle. L'objectif de cette étude est d'anlayser les contraintes d'accès aux soins de santé maternelle dans la ville de Maroua. L'étude a été éffective en adoptant une demarche hypothético-déductive basée sur la collecte et le traitement des données. Des enquêtes ont été menées auprès de 382 femmes en âge de procréer dans 19 aires de santé de la ville de Maroua. Les resultats montrent que, l'âge, la parité, le niveau d'instruction, le statut matrimonial et la religion sont les contraintes socio- culturelles qui limitent l'accès aux soins de santé maternelle. De même, le coût élévé du transport et des prestations, la distance et le lieu de résidence par rapport à la structure sanitaire constituent les contraintes économiques et spatiales.Toutes ces contraintes entravent ainsi la bonne prise en charge des femmes dans la mesure où les impacts sur la satisfaction de leurs demandes de soins de santé sont réels.
\end{abstract}

Mots clés: Contraintes, accès aux soins, santé maternelle, Maroua, Extrême-Nord

Date of Submission: 25 November $2016 \longrightarrow$ Date of Accepted: 30 January 2017

\section{INTRODUCTION}

La santé maternelle, dans le monde en général et dans les pays en développement en particulier, est au cœur des politiques nationales et internationales du secteur sanitaire. En effet, dans les pays d'Afrique, la mortalité maternelle est l'une des causes recurrentes de decès des femmes et des nouveaux-nés. Depuis quelques décenies, des mesures de réduction de la mortalité maternelle sont appliquées dans de nombreux pays. Cela a entrainé une baisse du taux de mortalité maternelle qui est passé de 870 en 1990 à 630 décès pour 100000 naissances vivantes en 2008 soit une reduction remarquable de 27\% entre 1990 à 2010 (Tekam, 2013). Une étude conjointe ménée par l'Unicef, l'OMS, l'UNFPA et la Banque Mondiale a déterminé que les décès maternels en Afrique pourraient baisser de $70 \%$ et les décès de nouveaux nés dimunier de près de la moitié, si le monde doublait l'investissement dans la planification familiale et les soins liés à la grossesse. À cet effet, de nombreuses initiatives et programmes internationaux en rapport avec la santé maternelle, notamment l'initiative pour une maternité sans risque, les Objectifs du Millénaire pour le Developpement, ont été mises sur pied. En dépit de tous ces projets, la mortalité maternelle est toujours effective dans la plupart des pays en développement notamment au Cameroun. Selon l'enquête démographique et de santé (EDS 2005) au Cameroun, le ratio de mortalité maternelle est de 762 décès pour 100000 naissances vivantes (EDS 2011). Un taux qui reste encore largement élévé et laisse entrevoir l'incapacité du pays à atteindre l'OMD $\mathrm{N}^{\circ} 5$ qui était celui de réduire la mortalité maternelle de $3 / 4$ en 2015. Plusieurs facteurs expliquent cette situation parmi lesquels le faible recours des femmes aux services de santé maternelle dans la majorité des villes du pays et particulièrement celles de l'Extrême-Nord. En effet, dans la plupart des villes camerounaises et particulièrement dans la ville de Maroua, la santé maternelle est fragilisée à cause du faible recours des femmes aux soins de santé maternelle. La proportion des femmes qui reçoivent les soins prénataux d'un agent de santé est de $59 \%$ dans l'Extrême-Nord, $64 \%$ dans le Nord et $72 \%$ dans la région de l'Adamaoua.Dans les régions méridionales par contre, cette proportion est très élévée avec $96 \%$ à l'Ouest, $98 \%$ dans le Nord-Ouest et $99 \%$ dans le Littoral(EDS 2005). Ces inégalités socio-spatiales en matière de santé et de recours aux soins sont largement reconnues aujourd'hui du fait que toutes les femmes n'ont pas accès aux mêmes services de santé.Toutefois, il est important de souligner que l'accès aux soins de santé n'est pas uniquement tributaire de l'offre à savoir, la qualité du service offert mais aussi et surtout de la demande de service qui, elle aussi, est très variable, selon de nombreux facteurs 
socio-économiques et spatiaux. La ville de Maroua dispose de quelques structures sanitaires de qualité, mais on enregistre une faible fréquentation des femmes dans ces services. Cette situation est due aux contraintes socioéconomiques et de mobilité qui limitent l'accès des femmes aux soins de santé maternelle. C'est dans l'optique d'une meilleure compréhension des inégalités et des difficultés d'accès aux soins de santé maternelle que nous nous interessons à la thématique portant sur les contraintes d'accès aux soins de santé maternelle dans la ville de Maroua.

\section{CONTEXTE SCIENTIFIQUE}

La problématique de la santé en général et de la santé maternelle en particulier interesse, à plus d'un titre, de nombreux auteurs d'où l'existence de nombreux travaux de recherches, articles et ouvrages sur les questions de santé dans le Monde.

La question d'accessibilité aux structures sanitaires a fait l'objet de plusieurs recherches. Cisse B. (2002) affirme que «l'accès aux soins de santé pour la plupart des cas en Afrique subsaharienne dépend, non seulement, de l'état de santé tel que perçu par l'individu, mais également des facteurs économiques tels que le revenu, le prix des services et des biens et l'accessibilité géographique en termes de distance, moyens et coût de transport qui séparent le patient du centre de santé ». Dans le même ordre d'idées, Gérard C. (2001), affirme qu'au Cameroun, « la difficulté d'accès aux soins de base est due, soit à l'éloignement physique, soit aux facteurs liés à la qualité du service ». Pour apporter plus d'explication aux disparités d'accès aux soins de santé Beninguisse (2001) se fonde sur l'anthropologie en se basant sur une approche purement culturelle. Cette approche met en relation la fréquentation des services de santé avec les institutions sociales telles que la religion, et permet ainsi, d'appréhender les comportements des femmes vis-à-vis du système de soins pendant les différentes étapes relatives à leur maternité.

Une autre approche intéressante aborde la question en mettant en avant les aspects liés à l'offre de soins de santé. Il s'agit de l'approche Biomédicale. Elle explique la faible fréquentation des services de soins maternelle surtout dans les pays émergents par l'insuffisance quantitative et qualitative des services de soins de santé, leur faible accessibilité financière (coûts prohibitifs de consultation et traitement) et géographique (éloignement des services). En effet, la rationalité qui sous-tend cette approche est que «l'offre crée sa propre demande». Autrement dit, l'utilisation des services de santé maternelle dépend d'abord de l'existence de ceux-ci ; ce qui semble ne pas refléter les réalités sur le terrain. Enfin, Les études démographiques ont l'avantage de mettre en relief les principaux obstacles en matière de recours aux soins pendant la grossesse, l'accouchement et le postpartum. Elles mettent en relation l'utilisation des services de santé maternelle avec les caractéristiques sociodémographiques telles que l'âge, la parité atteinte, l'éducation, le niveau de vie, l'affiliation religieuse, la taille et la structure du ménage.

À travers cette synthèse, l'on se rend compte que le recours aux soins pendant la grossesse dépend des caractéristiques socio-culturelles, socio- démographiques et économiques de la femme ainsi que du contexte institutionnel.

À ces facteurs socio-culturels et démographiques s'ajoutent d'autres facteurs explicatifs des disparités d'accessibilité aux soins de santé. Diverses études considèrent l'accessibilité géographique comme un facteur limitant majeur de l'utilisation des services des soins. Une étude réalisée par le CRDI entre 1994 et 1995 a révélé que l'éloignement du centre de santé avait une forte influence sur la fréquence des CPN au deuxième et au troisième trimestre de la grossesse. "Les femmes sont frustrées et découragées lorsqu'elles parcourent de longues distances à pieds pour se rendre au centre de santé et se font dire que le travailleur de la santé n'est pas là, qu'une seule personne est disponible, qu'elle doit s'occuper de beaucoup de gens et qu'elle ne peut donc pas prêter l'attention nécessaire ni offrir un service personnalisé à chacun des clients »(CRDI, 1995).

\section{Délimitation spatiale}

La présente étude est menée dans la ville de Maroua, chef-lieu du département du Diamaré dans la région de l'Extrême-Nord Cameroun. Elle est située entre $10^{\circ} 35$ de latitude Nord et $14^{\circ} 19$ de longitude Est. Elle est subdivisée en trois arrondissements à savoir Maroua I, Maroua II et Maroua III (Figure 1). 


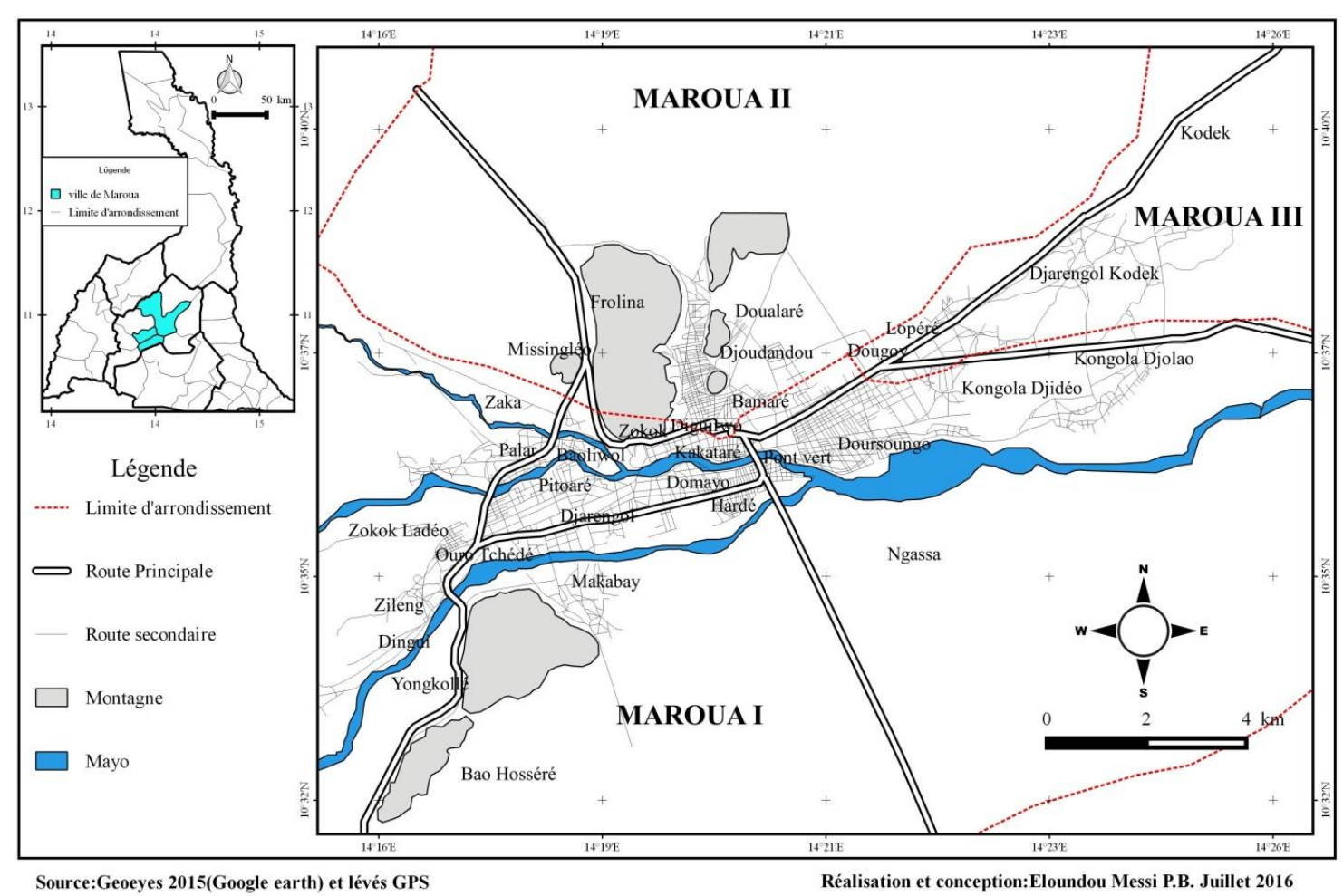

Figure1. Carte de localisation de la zone d'étude

\section{METHODOLOGIE}

L'analyse spatiale des inégalités de santé des populations et la question d'accessibilité aux soins de santé en tant que spécialité de la géographie humaine interagit avec d'autres champs et disciplines scientifiques. Les différentes notions empruntées et les façons d'objectiver le raisonnement scientifique peuvent donc être issues de nombreuses autres disciplines (économie, sociologie, statistique, écologie..), dans le souci d'une meilleure description qualitative et quantitative. Ainsi, pour l'atteinte des objectifs fixés à cette étude, nous avons procédé à la collecte des données et à leurs traitements grâce à divers outils d'analyse issus de diverses disciplines.

\section{Collecte des données}

Afin de prendre connaissance des travaux antérieurs concernant la question des disparités socio-spatiales d'accès aux soins de santé maternelle au Cameroun en général et dans la ville de Maroua en particulier, les données secondaires ont été obtenues grâce à la documentation écrite constituée de livres, d'articles, de revues, de données administratives, de sources électroniques, de mémoires, de thèses, de registres de consultations prénatales.

Les données primaires, quant à elles, ont été obtenues à partir de divers procédés de collecte à savoir :

-l'observation qui a consisté à faire des sorties sur le terrain pour nous imprégner des réalités de la zone d'étude et identifier les différentes formations sanitaires qui ont fait l'objet de cette étude.

-Entretiens : ils étaient menés avec les chefs des services de santé de la maternité de Maroua urbain, afin d'avoir les données démographiques des femmes en âge de procréer (FAP) des différentes formations sanitaires de la ville, le niveau d'équipement de ces établissements, l'effectif et les profils des personnels soignants et les types des soins offerts aux femmes en âge de procréer, leur calendrier de consultations prénatales (CPN) et de vaccination, d'avoir une idée sur les coûts de prestation des services offerts aux femmes. Ces entretiens ont été effectués à l'aide d'un guide d'entretien. Les informations obtenues ont permis de montrer l'inégale répartition des structures sanitaires et des personnels soignants, de calculer les différents ratios par exemple le nombre de patients par infirmier accoucheur, par sage-femme et gynécologue afin de faire la comparaison entre les arrondissements de Maroua urbain pour mesurer et réajuster l'offre et la demande des patientes.

-Echantillonnage : Pour cette étude, nous nous sommes intéressés aux femmes en âge de procréer (13-50 ans) des différentes aires de santé de Maroua urbain et nous avons obtenu un échantillonnage de 382 femmes issues des 19 aires de santé et reparties dans le tableau I ci-dessous. 
Tableau I. Échantillonnage des femmes enquêtées

\begin{tabular}{|l|l|l|l|}
\hline Aire de santé & Femme en âge de procréer (F.A.P) & Fréquence & Échantillon par formation sanitaire \\
\hline Brio & 410 & 0,68963197 & 3 \\
\hline Djarengol Kodek & 5134 & 8,63553791 & 33 \\
\hline DomayoDjarma & 4136 & 6,95687277 & 27 \\
\hline Domayo II & 3229 & 5,43127229 & 21 \\
\hline DomayoKaïgama & 1849 & 3,11007199 & 12 \\
\hline Doualaré & 8697 & 14,628608 & 56 \\
\hline Douggoi & 7673 & 12,9062101 & 49 \\
\hline Founangué & 4088 & 6,87613537 & 26 \\
\hline Kodek & 1649 & 2,77366615 & 11 \\
\hline Lopéré & 9645 & 16,2231716 & 62 \\
\hline Makabaye & 2187 & 3,67859786 & 13 \\
\hline Ngassa & 519 & 0,87297315 & 3 \\
\hline Ouro-Tchédé & 2501 & 4,20675503 & 16 \\
\hline Palar & 2596 & 4,3665478 & 17 \\
\hline Zokok & 5139 & 8,64394806 & 33 \\
\hline Total & 59452 & 100 & 382 \\
\hline
\end{tabular}

Source : Enquêtes de terrain, 2015 données de la Délégation Régionale de la santé de l'Extrême-Nord

\section{Traitement des données}

Les données obtenues ont été soumises à un traitement qualitatif et quantitatif. Le traitement quantitatif des données de terrain est basé sur des informations chiffrées issues du dénombrement des questionnaires d'enquête à travers le dépouillement. Ce traitement mené de manière quantitative et qualitative a été possible grâce aux logiciels statistiques Excel 2010 et SPSS 22 et a abouti à la réalisation des diagrammes.

\section{Les contraintes socio-culturelles}

\section{RÉSULTATS}

L'accès au soins de santé maternelle est influencé par les contraintes socio-culturelles principalement

l'âge, la parité, le niveau d'instruction, le statut matrimonial, la taille du ménage et la réligion.

\section{L'âge et la parité}

Boubacar Sow (1994) a démontré dans une étude que l'âge de l'individu agit négativement sur l'accès aux soins. Ce constat est bien visible dans les soins prénataux. En effet, le taux d'accès aux soins prénataux est faible pour le cas des jeunes femmes et celles en âge avancé. Par contre, pour celles ayant un âge moyen, le taux d'accès est élevé.

S'agissant de la parité, il ressort que c'est un déterminant significatif de l'utilisation des services de santé. Elle peut, en effet, influencer le recours aux soins prénataux. Les femmes qui ont peu ou pas encore d'enfants ont difficilement recours aux soins prénataux. Celles qui sont à leur première grossesse recourent le plus aux soins ; elles représentent $40 \%$ de l'ensemble des femmes qui ont effectué la visite prénatale contre $34,17 \%$ pour celles ayant deux à trois enfants, $22,5 \%$ pour celles ayant 4 à 5 enfants et 3,33\% pour les femmes ayant fait 6 accouchements ou plus (Figure 2). En effet, les femmes multipares fréquentent moins les services de soins obstétricaux parce que les expériences de grossesses antérieures les amènent à penser qu'elles peuvent s'en passer.

$\begin{array}{rccc}70 & & & \\ 60 & 34 & 23 & \\ 50 & 40 & & 3 \\ 40 & & & \text { Multipare(4-5) } \\ 30 & & & \begin{array}{c}\text { Grand Multipare (6 } \\ \text { à plus) }\end{array} \\ 20 & & & \end{array}$

Source : Enquêtes de terrain, 2015

Figure 2 . Les parités des gestantes enquêtées 
La courbe de tendance ajoutée à cette figure montre que les consultations prénatales décroissent au fur et à mesure que le nombre d'enfants des gestantes augmente. Le taux de consultation décroit donc allant de $40 \%$ pour les primipares à $3,33 \%$ pour les grandes multipares. La multiparité constitue donc de ce fait un frein au recours aux soins prénataux. Ainsi on peut conclure que le nombre de grossesses influe sur l'accès aux soins de santé.

\subsection{Le statut matrimonial et la religion}

Le statut matrimonial a une incidence sur la fréquentation des services de santé maternelle pendant la grossesse et l'accouchement. Les femmes célibataires disposent rarement des ressources nécessaires pour accéder aux soins modernes, car elles ont tendance à avoir un suivi prénatal moins adéquat que les autres femmes à cause du caractère non désiré de la grossesse et par conséquent, moins enclines à effectuer une visite prénatale. Par contre, les femmes mariées font plus de visites d'autant plus qu'elles ont l'apport financier de leurs conjoints pour des soins obstétricaux continus.

La religion influence aussi considérablement l'accès aux soins de santé maternelle dans la ville de Maroua. En effet, certaines pratiques religieuses s'inscrivent directement dans la vie sociale : l'habillement, l'alimentation, le recours aux soins de santé modernes. Dans la ville de Maroua, il existe trois principales religions qui sont (le Christianisme, l'Animisme et l'Islamisme) généralement associées à une diversité de modèles de perceptions du recours aux soins de santé modernes. Il en découle un ensemble de pratiques du système de santé traditionnel propre à chaque religion. C'est pourquoi la prise en charge des grossesses et des accouchements est susceptible d'être influencée par l'appartenance à une obédience religieuse. Il ressort de nos analyses que près de $41 \%$ des femmes vivant dans des ménages d'obédience catholique ou protestante ont accès régulièrement aux soins de santé. Par contre, on note seulement $31 \%$ des femmes musulmanes et $28 \%$ des femmes animistes.

$28 \%$


4 Christianisme
$41 \%$

Islam

$31 \%$

Source : Enquêtes de terrain, 2015

Figure 3. Répartition des femmes selon leur religion

La conclusion qui se dégage de cette analyse est que le christianisme confère des comportements favorables à un suivi de la grossesse et de l'accouchement contrairement à l'islam ou les femmes sont beaucoup contraintes à rester dans leur concession, attendant le jour de l'accouchement. Certaines femmes musulmanes pauvres préfèrent accoucher chez les accoucheuses traditionnelles pour ne pas montrer leur nudité aux inconnus qui peuvent être le personnel sanitaire de sexe masculin.

\subsection{Le niveau d'instruction}

Le niveau d'instruction joue un rôle fondamental dans l'explication des comportements des hommes dans la société. Plus le niveau d'instruction de la femme est bas, plus le recours aux soins de santé moderne est moindre voire inexistant. Ainsi, l'accès aux services de santé maternelle augmente avec le niveau d'instruction de la femme. Par conséquent, le manque d'instruction constitue un obstacle à la prise en charge de la grossesse et à la continuité des soins de santé prénataux. Sur 50 gestantes ayant fait quatre (4) CPN, $50 \%$ ont le niveau supérieur et $42,86 \%$ le niveau secondaire contre $7,14 \%$ le niveau primaire et $0 \%$ pour celle qui n'ont aucun niveau. Nous pouvons dire que le résultat de notre enquête montre que $60 \%$ des femmes en gestation qui pratiquent l'islam ne rendent pas dans les formations sanitaires modernes. 


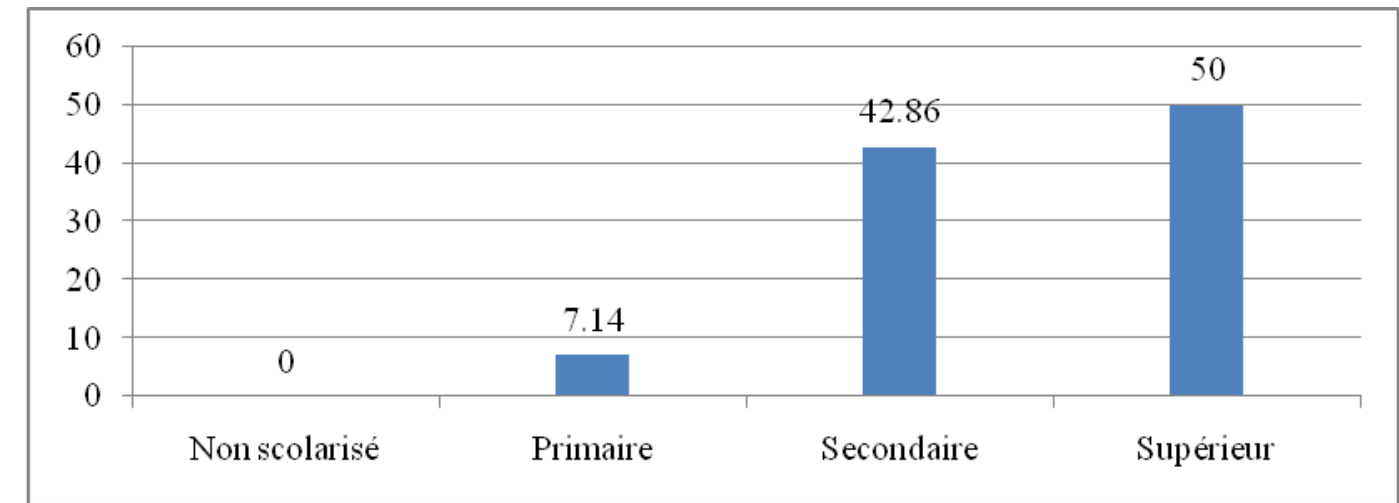

Source : Enquêtes de terrain 2015

Figure. 4 Repartition par niveau d'instruction des gestantes

Pour ce qui est de la taille du ménage, il ressort que l'accès aux soins de santé diminue avec l'augmentation de la taille du ménage. Cela signifie que si le revenu d'un ménage est réparti équitablement parmi les membres du ménage, ceux appartenant à des ménages de grande taille sont relativement désavantagées par rapport à ceux de ménages petite taille, quelle que soit la situation socio-économique du ménage. La figure 5 ci-dessous illustre à juste titre cette situation.

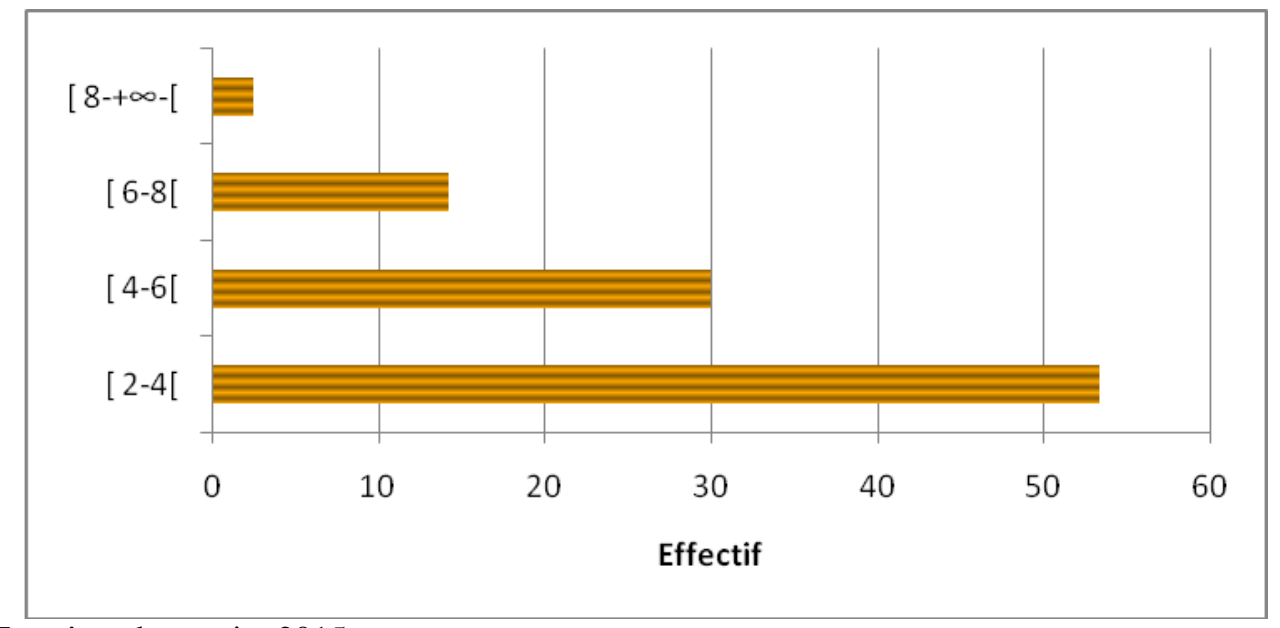

Source : Enquêtes de terrain, 2015

Figure 5. Taille du ménage des femmes enquêtées

Nous constatons à partir de cette figure 5 que, chez les enquêtées dont la taille du ménage se situe entre (2 et 4$)$ personnes représentent $83,33 \%$ qui ont utilisé les services des soins tandis que celles dont la taille est supérieure ou égale à 6 représentent $16,67 \%$. Car les besoins familiaux y compris les soins de santé augmentent avec la taille du ménage.

\section{2-Contraintes économiques}

\subsection{La profession et le faible revenu des gestantes}

Le statut social et le niveau de vie des gestantes ont une grande influence sur l'accès aux soins de santé maternelle. En effet, les femmes qui n'exercent aucune profession ont des difficultés pour avoir accès aux formations sanitaires. Celles qui ont un travail formel, un revenu et peuvent par contre, avoir un suivi de la grossesse dans une formation sanitaire. Dans la ville de Maroua, il ressort que les femmes n'exerçant aucune fonction et ayant un revenu bas font moins ou presque pas les quatre CPN tel que illustré par la figure 6 suivante. 


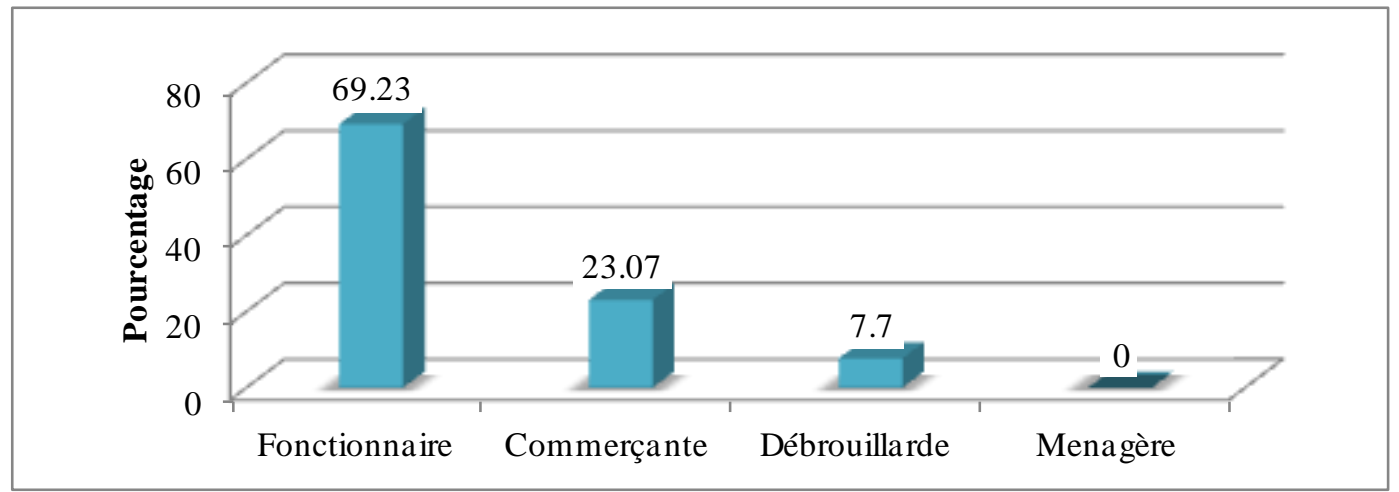

Source : Enquêtes de terrain 2015

Figure 6. Proportion des femmes faisant $4 \mathrm{CPN}$ selon leur statut social

\subsection{Coûts élevés de transports pour la formation sanitaire}

L'accessibilité aux soins se mesure par le pourcentage de la population pouvant se rendre à un centre de santé en moins d'une heure de marche ou en empruntant un moyen de transport facilement disponible (Monekosso, 1993). Les moyens de déplacement utilisés par les gestantes de Maroua urbain pour accéder aux services sanitaires sont entre autres la marche à pied, la moto et la voiture. Mais le coût élevé de ce moyen de déplacement pour atteindre une structure sanitaire est un facteur limitant l'accès aux soins de santé maternelle. Car plus le coût de déplacements est élevé, plus le taux de fréquentation des structures sanitaires diminue comme l'indique la figure 7.

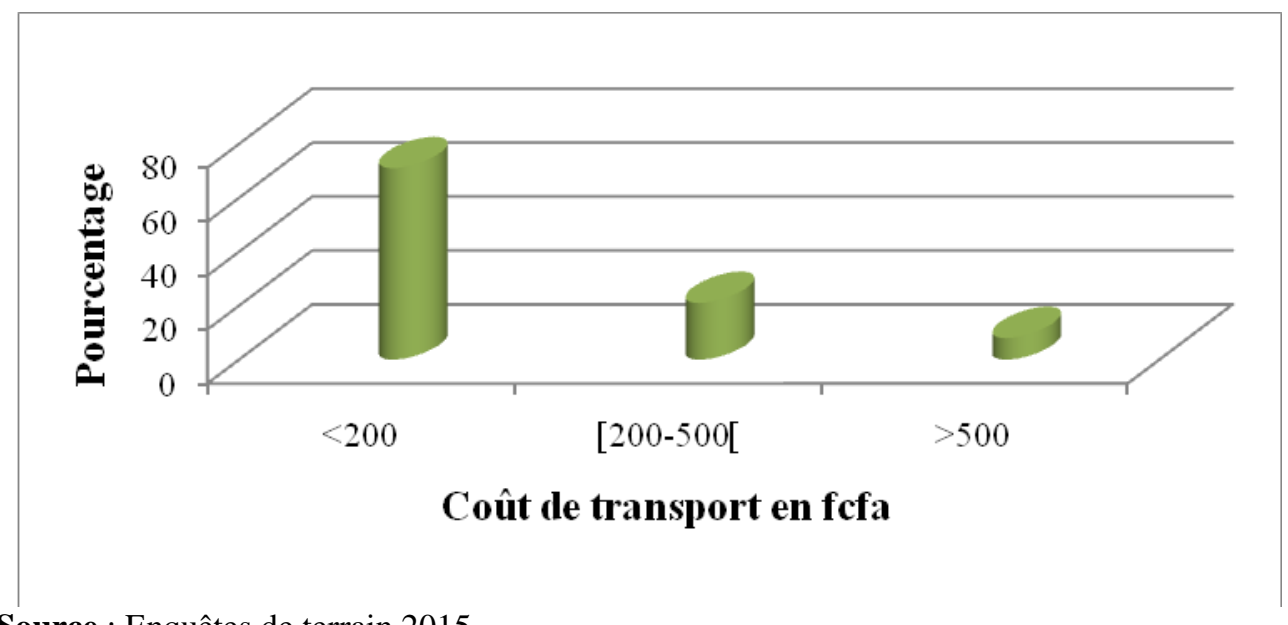

Source : Enquêtes de terrain 2015

Figure 7. La répartition des coûts de moyens de déplacement vers les formations sanitaires

Il ressort à partir de cette figure 7 que plus le coût de déplacement augmente plus le taux de fréquentation diminue. Donc le coût élevé du transport limite l'accès aux soins de santé maternelle.

\subsection{Coûts élevés de prestation de service}

Les coûts élevés des soins de santé sont susceptibles de constituer un facteur limitant l'accès aux soins de santé maternelle. En effet, lorsque le coût de soins est élevé et que la patiente ne dispose pas de moyens nécessaires, elle recourt à d'autres alternatives qu'elle trouve à la portée de sa bourse pour se faire soigner ou pour ne pas se faire soigner du tout. Par ailleurs, en raison des coûts d'accès et de prise en charge relativement élevés dans les structures sanitaires privées à but lucratif, les formations sanitaires publiques et privées à but non lucratif (confessionnelles en général) et public sont les plus sollicitées.

\section{Contraintes spatiales}

\subsection{L' éloignement par rapport au centre de santé}

Les travaux antérieurs qui se sont intéressés à l'effet de la distance sur l'utilisation des services de santé mettent en évidence une diminution des taux d'utilisation avec l'augmentation de la distance. La distance qui 
sépare le domicile d'une gestante et le centre de santé est importante pour l'accès aux soins de santé maternelle. Il ressort que des probabilités sont plus élevées chez les femmes résidant à moins de $5 \mathrm{~km}$ que chez celles qui résident à plus de $5 \mathrm{~km}$ des centres de santé pour faire la CPN dans une formation sanitaire.

Tableau II. Répartition de nombres de CPN faites par les enquêtées en fonction dela distance

\begin{tabular}{|l|l|l|l|l|}
\hline Distance & $(\mathbf{0 - 5})$ & $\mathbf{( 5 - 1 0 )}$ & $\mathbf{( 1 0 - 1 5 )}$ & Total \\
\hline 0 CPN & 2 & 14 & 0 & 16 \\
\hline $\mathbf{1 ~ C P N}$ & 92 & 22 & 0 & 114 \\
\hline $\mathbf{2}$ CPN & 94 & 13 & 0 & 107 \\
\hline 3CPN & 87 & 8 & 0 & 95 \\
\hline 4 CPN & 47 & 3 & 0 & 50 \\
\hline Total & 322 & 60 & 0 & 382 \\
\hline
\end{tabular}

Source : Enquêtes de terrain, 2015

En effet, il ressort de notre analyse que sur les 16 femmes n'ayant pas fait la CPN, environ 0,5\% résident à moins de $5 \mathrm{~km}$ des formations sanitaires contre $3,7 \%$ qui habitent à plus de $5 \mathrm{~km}$ de la formation sanitaire la plus proche. En effet, on constate que, plus la distance est grande, plus la structure sanitaire est de moins en moins fréquentée par les femmes en gestation. Les moyens de transport de nombreuses familles dans la ville de Maroua ne permettent pas souvent aux chefs de familles de s'acquitter aisément de ces tâches familiales, alors vient s'ajouter le problème de naissance d'un nouveau-né en ce temps où le phénomène de l'insécurité bat son plein avec la secte islamique Boko-Haram, les déplacements sont très limités de jour comme de nuit.

\subsection{Le lieu de résidence}

Le lieu de résidence a un impact significatif sur l'accès aux soins de santé. Le fait de résider dans la zone périurbain agit négativement sur le recours aux soins, puisque les structures sanitaires situées en périphérie sont sous équipées en matériel mais aussi en personnels de santé. Concernant l'accessibilité physique, notamment le lieu de résidence, nous constatons qu'en plein centre-ville, les femmes ont plus tendance à accoucher dans une formation sanitaire que celles qui résident en zone périphérique. Il est donc très difficile, pour une femme résident à plus de $5 \mathrm{~km}$ de la structure sanitaire, d'avoir recours à cette dernière pour le suivi de la grossesse et l'accouchement. Les résidences en périphéries limitent donc l'accès aux soins de santé Maternelle.

\section{DISCUSSION}

La bonne santé des femmes dans le monde dépend en général du premier lieu du suivi des femmes et en particulier des femmes en gestation jusqu'à l'accouchement. Cependant, dans la plupart des pays en développement, ces épreuves restent encore fatales pour certaines qui perdent la vie durant ce processus. En effet, cette situation est due au fait que l'accès aux soins de santé maternelle devient contraignant malgré l'amélioration constatée dans certaines régions en développement (OMS, 1999). Les résultats montrent que les femmes ne bénéficient pas systématiquement d'un suivi durant leur grossesse ou d'une assistance au moment de l'accouchement à cause de divers obstacles qui limitent l'accès des femmes aux soins de santé. Dans la ville de Maroua, la religion a une grande influence sur l'accès aux soins de santé des femmes. Ce résultat est similaire à celui de Beninguisse (2001), qui met en relation la fréquentation des services de santé avec les institutions sociales telle que la religion et permet ainsi, de comprendre les comportements des femmes vis-à-vis du système de soins pendant les différents événements relatifs à la maternité. À côté de la contrainte culturelle, se greffent également les contraintes sociales, économiques et spatiales. Fournier et Haddad (1995), s'inspirant des travaux de Kroëger (1983) à cet effet, ont montré l'influence de ces trois contraintes sur la santé des femmes. Ainsi, l'on retrouve les caractéristiques individuelles prenant en compte les caractéristiques sociodémographiques telles que l'âge, le sexe, la taille du ménage, le nombre d'enfants, les attributs du chef de ménage et d'autres composantes, l'ethnie et la religion, le milieu de socialisation, l'occupation. Les caractéristiques communes concernent, quant à elles, les caractéristiques des services, la qualité, les coûts d'utilisation et l'accessibilité géographique.

\section{CONCLUSION}

De cette étude dont l'objectif était d'analyser les contraintes d'accès aux soins de santé maternelle, il ressort que les contraintes socio-culturelles, économiques et spatiales concourent à la limitation de l'accès aux soins de santé maternelle dans la ville de Maroua. S'agissant des contraintes socio-culturelles, nous relevons que l'âge, la parité, le statut, la religion, le niveau d'instruction et la taille du ménage sont autant de facteurs qui limitent l'accès aux soins de santé maternelle. Parmi les femmes enquêtées, nous avons constaté que les femmes dont l'âge est supérieur à 36 ans fréquentent moins les structures sanitaires, car elles représentent $13 \%$ contre celles dont l'âge est inférieur à 36 ans. En plus, on a noté que la religion est une contrainte d'accès aux soins de santé du fait que les animistes et les musulmanes fréquentent moins les formations sanitaires et représentent 
respectivement $28 \%$ et $31 \%$ contre $41 \%$ chez les chrétiennes. Pour ce qui est des contraintes économiques, il s'agit du faible revenu, le coût élevé du transport et de prestations. Enfin, pour les contraintes spatiales, l'on note que la distance et le lieu de résidence limitent l'accès aux soins de santé maternelle.

\section{BIBLIOGRAPHIE}

[1]. Adeline, L., 2011, Les difficultés d'accès aux soins de santé au Pakistan [en ligne]. Santé humanitaire au proche-orient, juillet 2011, n. 54, P 34-69. Disponible sur : <http://humanitaire.revues.org/1008>. Consulté le 29 avril 2015.

[2]. Angue, R., 2005, La discontinuité entre les soins prénatals et les soins à l'accouchement : Étude comparative entre le Gabon et le Cameroun, mémoire de DESS, Yaoundé, 2005, 73p.

[3]. Assemal, A., 2003, Les déterminants de la prise en charge médicale de la grossesse et de l'accouchement au Tchad, Mémoire de DESS, IFORD, Yaoundé, 2003, 93p.

[4]. Beninguisse, G., 2001, L'accessibilité culturelle : une exigence de la qualité des services et soins obstétricaux en Afrique. In IsiugoAbaniche, U.(éds.), Haddad, S., 1992, Utilisation des services de santé en pays en développement. In Claude,

[5]. Cissé, B., 2001, Poverty and Access to health care? Center of study African Economies (CSAE).Annual conference on understanding poverty and growth in sub-saharan Africa.http://abamako.com. consulté le 15 juillet 2016

[6]. CRDI., 2003, Obstacles à l'accès à de soins de qualité . [en ligne] Rapport sur la santé dans le monde. Disponible sur : <http://web.idrc.ca/fr/ev-27498-201-1-Do-Topic.html/188>. (Consulté le 18 Janvier 2015).

[7]. Djourdebbe, F., 2005, La déperdition des soins prénatals au Tchad, Mémoire de DESS, Yaoundé, 2005, 106p.

[8]. Gerard,C., Thomas,F., 2001, «Cohort reproductive patterns in low fertility countries », in population and developement review, $\mathrm{n}^{\circ} 1, \mathrm{P} .103-132$

[9]. Haddad, S., Nougtara, A., Ridda., 2004, Les inégalités d'accès aux services de santé et leurs déterminants au Burkina Faso, santé, société et solidarité, $\mathrm{N}^{\circ} 2$, décembre 2004, Vol. 55, pp. 97-103.

[10]. Moneret, B., 1993, L'accès aux soins des populations démunies, Paris, Harmattan, 177p.

[11]. Mormiche., 1995, L’accès aux soins : évolution des inégalités entre 1980 et 1991, Economie et statistique, ํo282, Mars 1995, Vol. 50, p. 3-19.

[12]. OMS., 2005, Donnons la chance à chaque mère et à chaque enfant. [en ligne]. Rapport sur la santé dans le monde Genève, avril 2005. Disponible sur: <http://www.who.int /whr//fr.>. Consulté le 14 avril 2015.

[13]. Picheral, H., 1984, La géographie de la santé. In Bailly, A. (éds.), Concepts de la géographie. Paris, Masson, pp. $173-180$.

[14]. Programme des Nations Unies pour le Développement (PNUD)., 2010, Que faut-il faire pour atteindre les objectifs du Millénaire pour le développement: une évaluation internationale [en ligne]. La campagne contre les fistules. Disponible sur: <http://www.fistules.org> Consulté le 14 Avril 2015.

[15]. Rapport Organisation Mondiale de la Santé (OMS)., 2010, les Objectifs du Millénaire pour le développement, [en ligne]. Base de données OMD de l'ONU. Disponible sur : (en ligne) <http://mdgs.un.org>, Consulté le 14 Avril 2015.

[16]. Tekam, F., 2013, Mortalité maternelle : Le Cameroun s'éloigne de l'Objectif du Millénaire pour le développement. (en ligne) <http://.Pressenza.com /fr/2013/06/Mortalité maternelle-le-cameroun—s'éloigne-de-l'objectif-du-millenaire-pour-ledeveloppement-omd/>.Consulté le 15juillet 2016

[17]. Tonnellier, F., 1996, Géographie de l'Offre de soins: tendances et inégalités. Données sociales, Credes, № 1117 , Juin 1996 , Vol. 45, pp 259-264. 